\title{
OPEN Specific inhibition of FGF5-induced cell proliferation by RNA aptamers
}

\begin{abstract}
Ryo Amano ${ }^{1}$, Masato Namekata ${ }^{2}$, Masataka Horiuchi ${ }^{3}$, Minami Saso ${ }^{1}$, Takuya Yanagisawa ${ }^{1}$, Yoichiro Tanaka ${ }^{4}$, Farhana Ishrat Ghani ${ }^{2}$, Masakuni Yamamoto ${ }^{2}$ \& Taiichi Sakamoto ${ }^{1 \bowtie}$

Fibroblast growth factor 5 (FGF5) is a crucial regulator of hair growth and an oncogenic factor in several human cancers. To generate FGF5 inhibitors, we performed Systematic Evolution of Ligands by EXponential enrichment and obtained novel RNA aptamers that have high affinity to human FGF5. These aptamers inhibited FGF5-induced cell proliferation, but did not inhibit FGF2-induced cell proliferation. Surface plasmon resonance demonstrated that one of the aptamers, F5f1, binds to FGF5 tightly $\left(K_{\mathrm{d}}=0.7 \pm 0.2 \mathrm{nM}\right)$, but did not fully to FGF1, FGF2, FGF4, FGF6, or FGFR1. Based on sequence and secondary structure similarities of the aptamers, we generated the truncated aptamer, F5f1_56, which has higher affinity $\left(K_{d}=0.118 \pm 0.003 \mathrm{nM}\right)$ than the original F5f1. Since the aptamers have high affinity and specificity to FGF5 and inhibit FGF5-induced cell proliferation, they may be candidates for therapeutic use with FGF5-related diseases or hair disorders.
\end{abstract}

The fibroblast growth factor (FGF) family is involved in a wide variety of processes including cell proliferation, differentiation, and migration ${ }^{1-5}$. The diverse biological functions of FGFs are induced by binding to their receptors (FGFR1, FGFR2, FGFR3, and FGFR4) with the cofactor heparin sulfate ${ }^{4}$. The intracellular tyrosine kinase domains of the receptors are activated by FGF binding, followed the activation of various signaling pathways. FGF stimulation induces proliferation, differentiation, and migration, and FGFs are therefore involved in angiogenesis and fibrosis in several diseases and tumor growth ${ }^{5}$.

FGF5 is a 268 -amino-acid protein $(29.1 \mathrm{kDa})$ that binds with high affinity to the IIIc isoforms of FGFR1 and FGFR2, which are preferentially expressed in mesenchymal lineages ${ }^{6,7}$. FGF5 was first identified by the screening of transforming oncogenes ${ }^{8,9}$ and later characterized as a major regulator of hair growth ${ }^{10-12}$; FGF5 also promotes angiogenesis of human aortic endothelial cells and spermatogonial stem cell proliferation ${ }^{13,14}$. Therefore, development of an FGF5-specific inhibitor may be value as a hair growth enhancer and for treatment of FGF5-induced cancers; this would also contribute to understanding the mechanism of FGF5-induced signal transduction.

Aptamers are short folded nucleic acids selected from large random sequence libraries by the process known as Systematic Evolution of Ligands by EXponential enrichment (SELEX) ${ }^{15,16}$. Aptamers have high affinity and specificity to their various target molecules and can be used as therapeutic agents ${ }^{17}$. For example, an RNA aptamer specific for FGF2 exerted a strong analgesic effect in a mouse model of bone cancer pain ${ }^{18}$, and an anti-FGF2 aptamer also inhibited the growth of FGF2-FGFR pathway driven lung cancer cells ${ }^{19}$. Furthermore, the antiFGF2 aptamer was examined for treatment of neovascular age-related macular degeneration (nAMD) ${ }^{20}$ and a clinical trial program has been conducted to assess the efficacy of the aptamer in patients with active nAMD.

Therefore, to develop therapeutic candidates for FGF5-related diseases or hair disorders, we performed SELEX and obtained anti-FGF5 aptamers that have high affinity and specificity to FGF5 and inhibit FGF5induced cell proliferation. This is the first report to characterize anti-FGF5 aptamers that inhibit FGF5-iduced cell proliferation.

\section{Results}

Selection and identification of RNA aptamers that bind to FGF5. We first obtained RNA aptamers against human FGF5 by SELEX. An initial pool of RNAs was transcribed from a template DNA containing 40 nucleotides of random sequence. To resist ribonuclease degradation, 2 '-fluoropyrimidine modifications were introduced to the RNA pool. The selection was performed against recombinant human FGF5 immobilized on nickel or cobalt charged resins. To select tightly binding aptamers, the resins were washed with buffer contain-

\footnotetext{
${ }^{1}$ Department of Life Science, Faculty of Advanced Engineering, Chiba Institute of Technology, 2-17-1 Tsudanuma, Narashino-shi, Chiba 275-0016, Japan. ${ }^{2}$ Advangen Inc, 4-6-3 Kashiwa, Kashiwa-shi, Chiba 277-0005, Japan. ${ }^{3}$ Faculty of Pharmaceutical Science, Health Sciences University of Hokkaido, 1757 Kanazawa, Toubetsu,

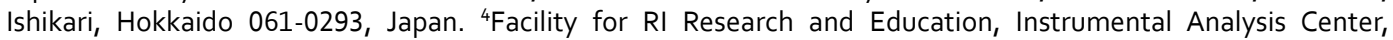
Research Initiatives and Promotion Organization, Yokohama National University, 79-5 Tokiwadai, hodogaya-ku, Yokohama 240-8501, Japan. ${ }^{\boxplus}$ email: taiichi.sakamoto@p.chibakoudai.jp
} 


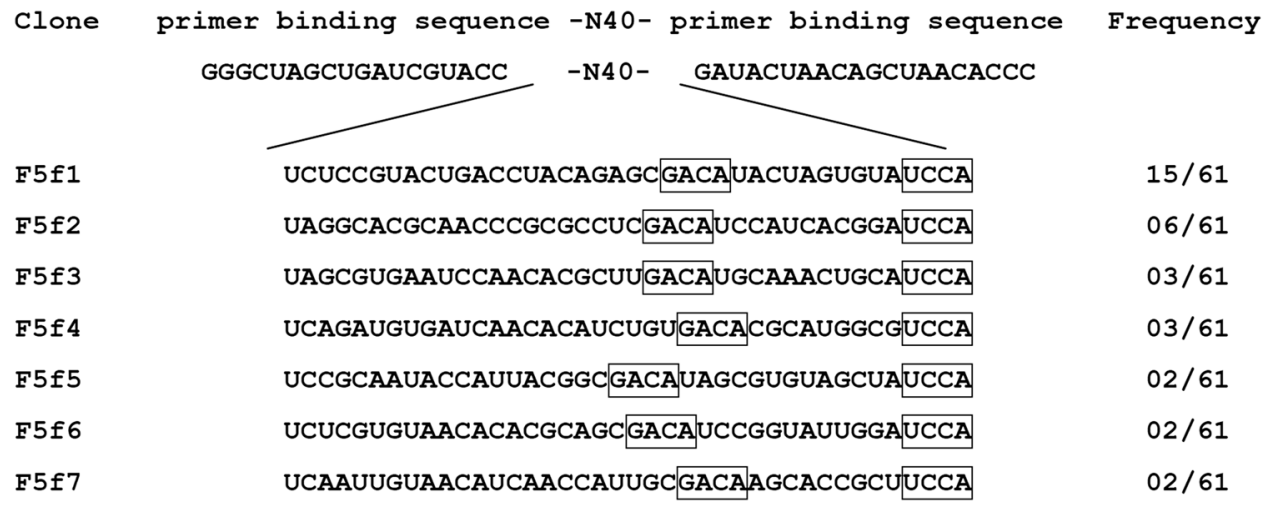

Figure 1. Sequence and frequency of the cloned aptamers. The selected sequences between primer binding sequences and their frequency among 61 clones are shown. The boxes indicate consensus sequences.

ing a high concentration of sodium chloride or urea with a low-molecular-weight heparin as the competitor. After seven rounds of SELEX, a total of 61 clones were randomly sequenced and seven unique sequences (F5f1F5f7) were successfully obtained (Fig. 1). Fifteen clones contained F5f1, which was the most frequently observed sequence (15/61). Furthermore, the aptamers from F5f1 to F5f7 contained the consensus sequences $5^{\prime}$-GACA-3' and $5^{\prime}-\mathrm{UCCA}-3^{\prime}$.

Specific inhibition of FGF5-induced cell proliferation by aptamers. We assessed the inhibition of FGF5 activity as well as specificity of aptamers using a NIH3T3 cell proliferation assay. FGFR1 is expressed in NIH3T3 cells; thus, exogenous FGF5 binds to FGFR1 to induce cell proliferation ${ }^{21}$. Addition of aptamers with 3 nM FGF5 to NIH3T3 cells resulted in the inhibition of cell proliferation in a concentration-dependent manner (Fig. 2a), which was not observed when random RNA was used. Aptamer concentrations at which $50 \%$ cell proliferation were inhibited ( $\mathrm{IC}_{50}$ value) were calculated (Table 1). The $\mathrm{IC}_{50}$ of the most frequently observed aptamer, F5f1, was $7.9 \pm 0.3 \mathrm{nM}$, whereas the aptamer with the highest inhibitory activity, F5f3, was $5.2 \pm 0.2 \mathrm{nM}$. On the other hand, the aptamers did not inhibit NIH3T3 cell proliferation when FGF2 was added to cells instead of FGF5. Moreover, we performed western blotting of phospho-FGFR1 and showed that the F5f1 aptamer inhibited FGFR1 phosphorylation in a dose-dependent manner, but not random RNA (Figs. 2c and S1). Therefore, the selected aptamers specifically inhibited the function of FGF5.

Binding affinity and specificity of aptamers against FGF5. We used surface plasmon resonance (SPR) to analyze the binding affinity of the F5f1 and F5f3 aptamers to FGF5. The dissociation constant $\left(K_{\mathrm{d}}\right)$ values of F5f1 and F5f3 binding to FGF5 were $0.7 \pm 0.2 \mathrm{nM}$ and $0.57 \pm 0.02 \mathrm{nM}$, respectively (Table 2 and Figure S2). The FGF5 specificity of F5f1 was confirmed by SPR analysis. F5f1 did not bind to FGF1, FGF2, FGF4, FGF6, or the extracellular domain of FGFR1 (Fig. 3). Furthermore, FGF5 did not bind to random RNA efficiently, although a small amount of nonspecific binding was observed (Figure S3). Similar to heparin, RNA is a highly negatively charged polymer and FGF5 has a positively charged heparin-binding site. Thus, weak binding of random RNA to FGF5 may be due to the nonspecific electrostatic interaction. Furthermore, we performed a competition assay and showed that F5f1 blocked the binding of FGF5 to FGFR1 (Fig. 4), consistent with the NIH3T3 cell proliferation assay results. Therefore, we revealed that the F5f1 aptamer specifically binds to FGF5 and competitively inhibits the binding of FGF5 to FGFR1.

Secondary structure prediction and truncation of F5f1 and F5f3 aptamers. We predicted the secondary structures of the aptamers to minimize and optimize the aptamers, using the CentroidFold program (http://rtools.cbrc.jp/centroidfold//22. The aptamers have a multi-branched loop that contains the consensus sequences 5'-GACA-3' and 5'-UCCA-3' (Fig. 5a, b). On the basis of the predicted secondary structure, F5f1_56 and F5f3_56 (Fig. 5c, d) with 56 nucleotides (nt) were generated from F5f1 and F5f3. Five GC base pairs were added to the truncated aptamers to stabilize the stem structure. The SPR-based $K_{\mathrm{d}}$ values of FGF5 binding to F5f1_56 and F5f3_56 were $0.118 \pm 0.003 \mathrm{nM}$ and $0.92 \pm 0.04 \mathrm{nM}$, respectively (Table 2 and Figure S2). Moreover, F5f1_56 and F5f3_56 inhibited NIH3T3 cell proliferation with $\mathrm{IC}_{50}$ values of $6.8 \pm 0.8 \mathrm{nM}$ and $8.2 \pm 1.4 \mathrm{nM}$, respectively (Fig. 2b). Thus, truncated variants retained the binding activity to FGF5 and inhibitory activity of NIH3T3 cell proliferation. Clements et al. estimated that the $K_{\mathrm{d}}$ for FGF5-FGFR1 is between 0.5 and $1.5 \mathrm{nM}$ according to the results of the competition assay with $\mathrm{FGF}^{23}$. The $K_{\mathrm{d}}$ and $\mathrm{IC}_{50}$ values of the aptamers are consistent with the estimated $K_{\mathrm{d}}$ for FGF5-FGFR1 in the previous study.

\section{Discussion}

In this work, we successfully obtained highly specific inhibitory RNA aptamers against FGF5. The aptamers from F5f1 to F5f7 had the consensus sequences 5'-GACA-3' and 5'-UCCA-3' (Fig. 1). Based on the predicted secondary structures of the aptamers, the consensus sequences were located in the multi-branched loop region, whereas the sequence of stem regions (Stems I and II) and apical loop regions (Loops I and II) varied (Fig. 5). This suggests 
a

F5f1

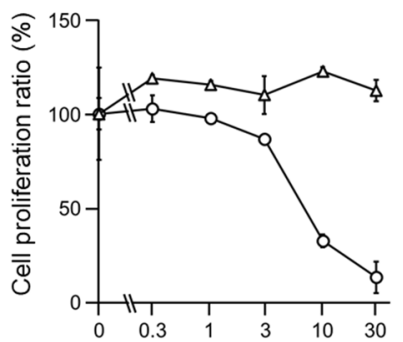

F5f5

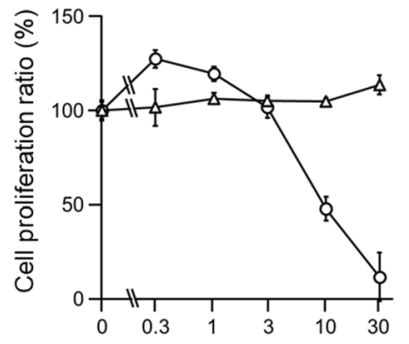

b

F5f1_56

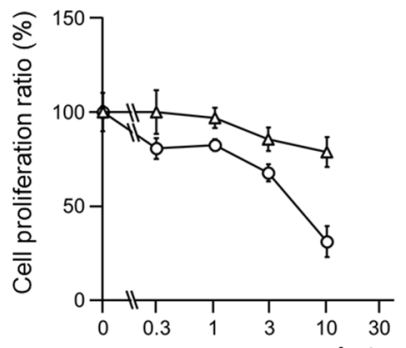

Aptamer (nM)
F5f2

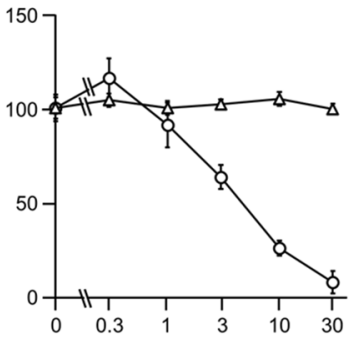

Aptamer (nM)
F5f3

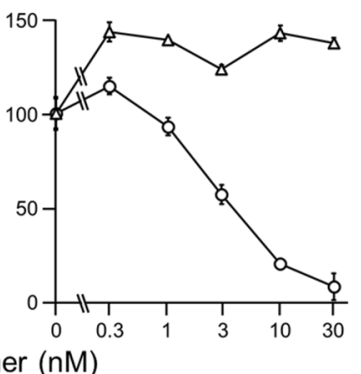

F5f7

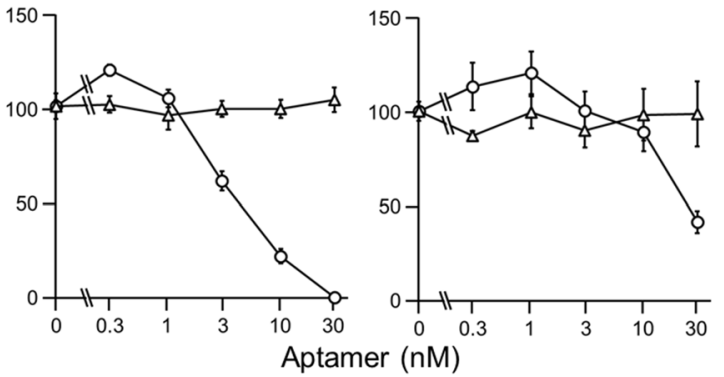

F5f4

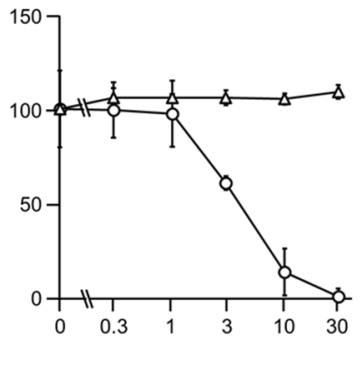

Random

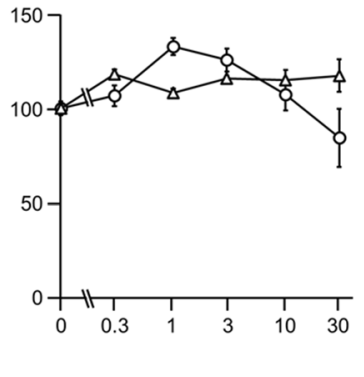

Figure 2. Inhibition of NIH3T3 cell proliferation by aptamers F5f1-F5f7 and random RNA (a) and the truncated aptamers F5f1_56 and F5f3_56 (b). Cells were cultured with human FGF5 ("circle") and human FGF2 ("triangle"). The experiments were performed three times, and the mean value and errors were shown. (c) Immunoblotting images of phospho-FGFR1 in FGF5-stimulated NIH3T3 cells in the presence of F5f1 (lanes 3-6) or random RNA (lanes 7-10).

\begin{tabular}{|l|c|}
\hline Clone & IC $_{\mathbf{5 0}}(\mathbf{n M})^{b}$ \\
\hline F5f1 & $7.9 \pm 0.3$ \\
\hline F5f2 & $6.2 \pm 1.7$ \\
\hline F5f3 & $5.2 \pm 0.2$ \\
\hline F5f4 & $6.0 \pm 2.4$ \\
\hline F5f5 & $12.4 \pm 0.2$ \\
\hline F5f6 & $5.7 \pm 0.2$ \\
\hline F5f7 & $27.8 \pm 0.1$ \\
\hline F5f1_56 & $6.8 \pm 0.8$ \\
\hline F5f3_56 & $8.2 \pm 1.4$ \\
\hline
\end{tabular}

Table 1. Aptamer inhibition of NIH3T3 cell proliferation ${ }^{a} .{ }^{a}$ The data of the cell proliferation assay are shown in Fig. $2 .{ }^{b} \mathrm{IC}_{50}$ is represented by the mean \pm standard error from three independent measurements. 


\begin{tabular}{|l|l|l|l|}
\hline Aptamer & $\boldsymbol{k}_{\text {on }}\left(\times \mathbf{1 0}^{\mathbf{5}} \mathbf{M}^{-1} \mathbf{s}^{-1}\right)$ & $\boldsymbol{k}_{\text {off }}\left(\times \mathbf{1 0}^{-5} \mathbf{s}^{-1}\right)$ & $\boldsymbol{K}_{\mathbf{d}}(\mathbf{n M})$ \\
\hline F5f1 & $3.3 \pm 0.3$ & $20 \pm 6$ & $0.7 \pm 0.2$ \\
\hline F5f3 & $1.7 \pm 0.3$ & $10 \pm 2$ & $0.57 \pm 0.02$ \\
\hline F5f1_56 & $1.8 \pm 0.3$ & $2.1 \pm 0.3$ & $0.118 \pm 0.003$ \\
\hline F5f3_56 & $1.36 \pm 0.05$ & $12.5 \pm 0.7$ & $0.92 \pm 0.04$ \\
\hline
\end{tabular}

Table 2. Kinetic parameters of aptamer binding to FGF5 ${ }^{a}$. ${ }^{a}$ The kinetic parameters are represented by the mean \pm standard error from three independent measurements.

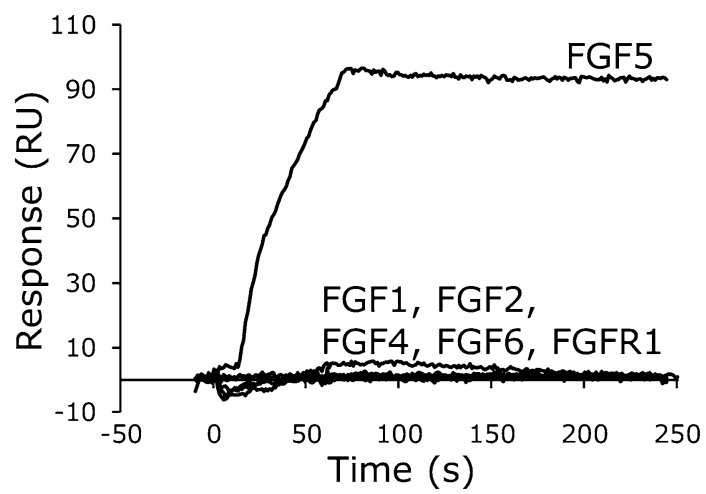

Figure 3. Specificity of F5f1 aptamer binding to FGF5. After immobilization of F5f1 aptamer on the sensor chip, 100 nM human FGF5, other human FGF family members, or extracellular domain of human FGFR1 was injected with four equivalents of low-molecular-weight heparin.

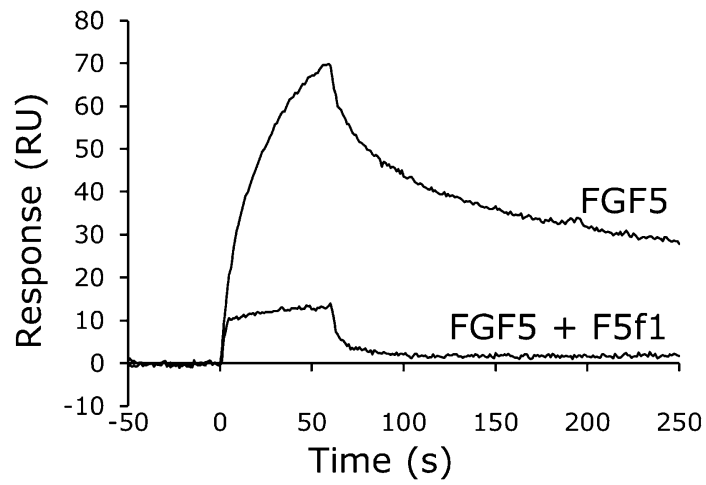

Figure 4. Competitive inhibition of the binding of FGF5 to FGFR1 by the F5f1 aptamer. After immobilization of the extracellular domain of human FGFR1 on the sensor chip, a mixture of 100 nM human FGF5 and $100 \mathrm{nM}$ low-molecular-weight heparin was injected with or without $200 \mathrm{nM}$ F5f1 aptamer.

that the multi-branched loop of aptamer is important for FGF5 binding. The flexible loop of aptamers is known to be important for induced fit to the surface of target proteins and binds to the proteins using weak interactions such as van der Waals contacts and hydrogen bonds ${ }^{24-27}$.

F5f1 aptamer bound to FGF5 with a high specificity as demonstrated by the cell proliferation assay and SPR (Figs. 2 and 3). The FGF family contains 22 identified members, which share sequence and structural similarity; therefore, it is important to ensure the specificity of the aptamer to avoid any side effects due to binding to the other FGF members. We confirmed that F5f1 did not bind to FGF4 and FGF6, which belong to the same subfamily as FGF5 in terms of sequence similarity (Figure S4). We further confirmed that the anti-FGF5 aptamers did not bind to FGF2. A clinical trial program using the anti-FGF2 aptamer has been conducted for active nAMD ${ }^{19}$; the sequence and secondary structure of the anti-FGF2 aptamer used in the previous study ${ }^{28}$ and our anti-FGF5 aptamers are different from each other (Figure S5). We also confirmed that F5f1 lacked affinity to FGFR1, which is the binding partner of FGF5. Aptamers that bind to FGFR1 or FGFR3 have been previously obtained ${ }^{29-31}$, and dimerized aptamers against FGFR1 or FGFR3 functioned as activators such as FGFs ${ }^{30,31}$. The sequences of these aptamers and our anti-FGF5 aptamers also differ from each other (Figure S5). Therefore, we expect that the anti-FGF5 aptamers will exhibit fewer side effects when used as therapeutic agents. 

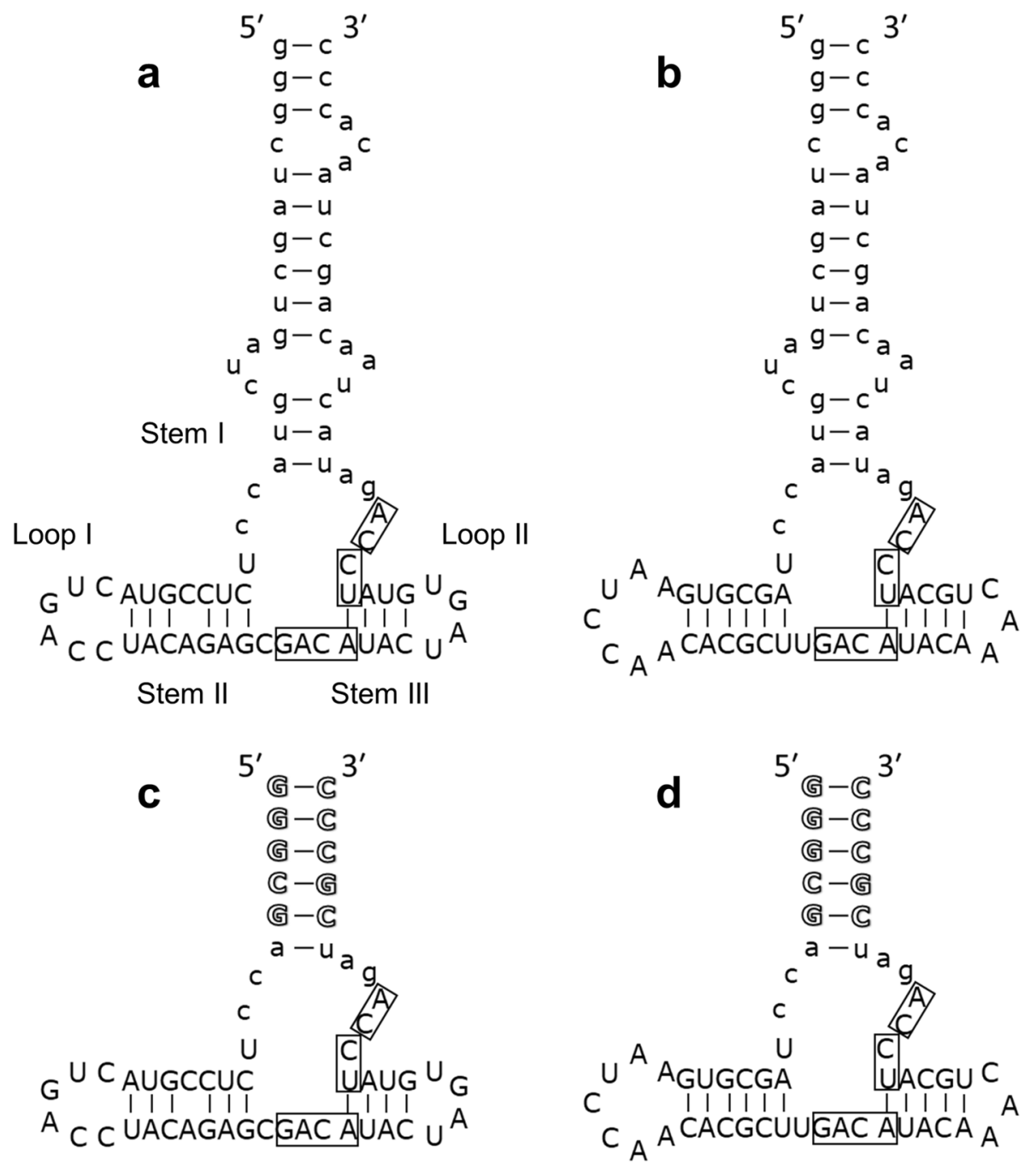

Figure 5. Predicted secondary structures of aptamers, F5f1 (a), F5f3 (b), F5f1_56 (c), and F5f3_56 (d). The consensus sequences are boxed. The lowercase and outline letters indicate the primer binding sequence and the added sequence, respectively.

FGF5 was originally identified as a transforming proto-oncogene ${ }^{8}$. Expression of FGF5 is increased in pancreatic cancer and associated with the occurrence and metastasis of pancreatic cancer ${ }^{32}$. Increased FGF5 expression was also observed in cell lines from renal cell carcinoma, prostate cancer, and breast cancer ${ }^{33}$, and overexpression of FGF5 in melanoma cells enhanced malignancy in vitro and in vivo ${ }^{34}$. Abnormal expression of FGF5 has been observed in non-small cell lung cancer (NSCLC) tissues. NSCLC cell lines exhibited high FGF5 expression and silencing of FGF5 in NSCLC cells inhibited cell proliferation and induced cell apoptosis ${ }^{35}$. FGF5 is also significantly upregulated in osteosarcoma (OS) tissues and cells. Knockout of FGF5 inhibited OS cell proliferation and tumor growth in a nude mouse model, and the addition of exogenous recombinant FGF5 to OS cells promoted cell proliferation while inhibiting cell apoptosis ${ }^{36}$. Moreover, FGF5 was identified as a direct target of the tumor suppressive microRNA ( $\mathrm{miR}$ ) miR-188-5p in hepatocellular carcinoma ${ }^{37}$; miR-567 also suppressed the cell proliferation and metastasis by targeting FGF5 in $\mathrm{OS}^{38}$. These studies indicate that FGF5 exerts oncogenic activity in several human cancer tissues and cells, and that anti-FGF5 aptamers might inhibit these FGF5-related cancers.

FGF5 is also known as one of the crucial regulators of the hair growth cycle ${ }^{10-12}$. This consists of three distinct sequential phases, anagen, catagen, and telogen. Anagen is the active phase of hair growth; catagen is the regression phase where hair elongation declines; and telogen is the resting phase where elongation stops completely and eventually progresses to hair loss. FGF5 is produced in the outer root sheath of the hair follicle in the late anagen dominantly and binds to FGFR1 of the dermal papilla cells, where it induces the transition from the anagen to catagen ${ }^{39,40}$.

Mutations in the FGF5 gene were identified from the angora phenotype in mice ${ }^{10}$ and trichomegaly in humans ${ }^{11}$, indicating that FGF5 terminates hair elongation consequently by switching from anagen to catagen. Therefore, inhibition of FGF5 activity by the aptamers may contribute to an extended anagen phase, resulting in promotion of hair growth and reduction of hair loss. In fact, a decapeptide showing selective inhibition of 
FGF5-binding with FGFR1 against FGF2-binding on NIH3T3 cells did recover coat hair growth that was suppressed by FGF5 administration in vivo ${ }^{21}$.

In this study, we obtained selected aptamers that specifically bind to FGF5 and do not bind to FGF1, FGF2, and subfamilies FGF4 and FGF6; these aptamers also inhibit FGF5-induced cell proliferation competitively. Furthermore, we succeeded in truncating the aptamer to 56 nucleotides. Therefore, these aptamers have the potential to be therapeutic agents for FGF5-associated cancers and hair loss.

\section{Materials and methods}

Construction of the expression plasmid for hFGF5. The DNA fragment coding residues 1-119 of Bombyx mori ß-1,3-glucan recognition protein (GRP) and the cleavage site of HRV 3C protease (3C) was amplified with polymerase chain reaction using KOD plus neo DNA polymerase (TOYOBO CO., LTD. Osaka, Japan) with the primers $5^{\prime}$-CATGCCATGGAGTACGAGGCACCACCGGC-3' and 5'-GGAATTCCATATGCGGGC CCTGAAACAGCACTTCCAGAAATTCTACTCCTGGTGTTATTTCAGAG-3' from pET-GRP-3C-His as a template ${ }^{41}$. The DNA fragment coding hFGF5 residues 21-242 [hFGF5 (21-242)] (GenBank id: NM_004464.3) was amplified with the primers 5'-CACCCATATGCACGGGGAGAAGCGTCTCG-3' and 5'-GCCCTCGAG AGGGCTAGGTGGCTTTTTCTTTTCAG-3' from Human Universal QUICK-Clone cDNA II (Takara Bio USA, Inc., CA, USA) as a template. DNA fragments of GRP-3C and hFGF5 (21-242) were cloned into pET-21d (+) (Merck KGaA, Darmstadt, Germany) to give pET-GRP-3C-hFGF5 (21-242)-His.

Construction of the expression plasmid for hFGFR1. The DNA fragment coding an artificial signal peptide Secrecon-AA ${ }^{42}$ was amplified using the primer $5^{\prime}$-GGTTTGGTGTTATCGGCGGCGGCC-3' from the oligonucleotide 5'-ATGTGGTGGCGCCTGTGGTGGCTGCTGCTGCTGCTGCTGCTGCTGTGGCCC ATGGTGTGGGCCGCCGCC-3'. The DNA fragment coding hFGFR1 residues 142-356 [hFGFR1 (142-356)] (GenBank ID NM_023110.2) was amplified using the primers 5'-GCCGCCGCCGATAACACCAAACCAAAC CGTATGCC-3' and 5'-CGCCTCCGCCCCTCTCTTCCAGGGCTTCCAG-3' from Human Universal QUICKClone cDNA II (Takara Bio USA, Inc.) as a template. The DNA fragment coding Gly-Gly-Gly-Gly-Ser-His-HisHis-His-His-His [G4S-His] was amplified using the primers 5'-CCTGGAAGAGAGGGGCGGAGGCGG-3' and 5'-GATCGAACCCTTTCAATGGTGATGGTGATGG-3' from the oligonucleotide 5'-GGCGGAGGCGGA AGCCTGGAGGTGCTGTTCCAGGGCCCCCACCATCACCATCACCATTGA-3' that was used as a template. The DNA fragment coding pcDNA was amplified using the primers $5^{\prime}$-CCATCACCATTGAAAGGGTTCGAT CCCTACC-3' and 5'-CGCCACCACATGGTGGTTCGATCCTCTAGAGT-3' from pcDNA3.4 plasmid that was used as a template (Thermo Fisher Scientific, Waltham, MA, USA). Three amplified DNA fragments of Secrecon-AA, hFGFR1 (142-356), and G4S-His were cloned into pcDNA using in vivo assembly cloning ${ }^{43}$. The resultant plasmid was named pcDNA-SecreconAA-hFGFR1 (142-356)-His.

Preparation of curdlan beads. Curdlan powder $(2.7 \mathrm{~g})$ (FUJIFILM Wako Pure Chemical Corp., Osaka, Japan) was dissolved in $270 \mathrm{~mL}$ of $0.6 \mathrm{M} \mathrm{NaOH}$ and centrifuged at $4670 \times g$ for $10 \mathrm{~min}$ at $25^{\circ} \mathrm{C}$. The soluble fraction was dispersed in $540 \mathrm{~mL}$ of 1-butanol at $1000 \mathrm{rpm}$ with Tornado laboratory high power mixer SM-101 with a stirring blade propeller (f50 mm) (AS ONE Co., Osaka, Japan); glacial acetic acid was added until curdlan beads formed while stirring. The curdlan bead suspension was filtered with stainless steel sieves (aperture of 100 and $150 \mu \mathrm{m})$ and curdlan beads with a particle diameter of $100 \sim 150 \mu \mathrm{m}$ were collected, suspended in trisbuffered saline (TBS) $(10 \mathrm{mM}$ Tris $\mathrm{HCl}, \mathrm{pH} 7.5,150 \mathrm{mM} \mathrm{NaCl})$ as a $50 \%$ slurry, and stored at $4{ }^{\circ} \mathrm{C}$.

Expression and purification of hFGF5. Escherichia coli BL21 (DE3) pLysS competent cells were transformed with expression plasmids pET-GRP-3C-hFGF5 (21-242)-His and the transformant was inoculated directly into $2 \times \mathrm{YT}$ medium containing $50 \mu \mathrm{g} / \mathrm{ml}$ carbenicillin and $34 \mu \mathrm{g} / \mathrm{mL}$ chloramphenicol, and incubated at $37^{\circ} \mathrm{C}$ at $120 \mathrm{rpm}$ until the absorbance at $600 \mathrm{~nm}$ was 1.3. Expression of GRP-3C-hFGF5 (21-242)-His protein was induced by addition of isopropyl $ß-\mathrm{D}$-1-thiogalactopyranoside to a final concentration of $0.1 \mathrm{mM}$. The culture was incubated at $16^{\circ} \mathrm{C}$ for $24 \mathrm{~h}$ with gentle agitation at $90 \mathrm{rpm}$. Cells were resuspended in lysis buffer ( $50 \mathrm{mM}$ sodium phosphate, $\mathrm{pH} 8.0,300 \mathrm{mM} \mathrm{NaCl}, 10 \mathrm{mM}$ imidazole, $5 \mathrm{mM} \mathrm{NaN}_{3}$ ) containing $1 \mathrm{mg} /$ $\mathrm{mL}$ lysozyme (Sigma-Aldrich Co. LLC., Missouri, USA) and disrupted using sonication two times on ice for 3 min (5-s pulse, 10-s pause, 80\% amplitude) using a Vibra-Cell Processor VCX-130 (Sonics \& Materials, Inc., Newtown, CT, USA) equipped with $6 \mathrm{~mm}$ probe; subsequently, $1 \%$ of Triton X-100 was added to the cell lysate. The cell lysate was cleared by centrifugation and loaded onto a Ni-NTA superflow (QIAGEN, Hilden, Germany) affinity column $(10 \mathrm{~mm}$ id $\times 100 \mathrm{~mm})$. The column was washed with 20 column volume of wash buffer $(50 \mathrm{mM}$ sodium phosphate, $\mathrm{pH}$ 8.0, $300 \mathrm{mM} \mathrm{NaCl}, 20 \mathrm{mM}$ imidazole, $5 \mathrm{mM} \mathrm{NaN}_{3}$ ) and GRP-3C-hFGF (21-242)-His was eluted with 5 column volume of elution buffer $(50 \mathrm{mM}$ sodium phosphate, $\mathrm{pH} 8.0,300 \mathrm{mM} \mathrm{NaCl}, 250 \mathrm{mM}$ imidazole, $5 \mathrm{mM} \mathrm{NaN}_{3}$ ). The eluate was mixed with the curdlan beads by a rotator for $1 \mathrm{~h}$ at $4{ }^{\circ} \mathrm{C}$. Complexes of GRP-tagged protein with curdlan beads were washed with 20 column volume of TBS and were treated with GST-HRV 3C protease for $35 \mathrm{~h}$ at $4{ }^{\circ} \mathrm{C}$ to release the hFGF5 (21-242)-His from the curdlan beads. The curdlan bead supernatant was loaded onto TOYOPEARL AF-Heparin HC-650 M (Tosoh Corp., Tokyo, Japan) affinity column $(10 \mathrm{~mm}$ id $\times 100 \mathrm{~mm})$. The column was washed with 20 column volume of $20 \mathrm{mM} \mathrm{HEPES}-\mathrm{NaOH}, \mathrm{pH}$ $7.5,300 \mathrm{mM} \mathrm{NaCl}$, and then hFGF5 (21-242)-His was eluted with a 20 column volume linear gradient from 300 to $3000 \mathrm{mM} \mathrm{NaCl}$ in $20 \mathrm{mM}$ HEPES-NaOH, pH 7.5. Fractions containing hFGF5 (21-242)-His protein were concentrated using ultrafiltration with the use of Amicon Ultra 4 centrifugal filtration device $(10 \mathrm{kD}$ molecular mass cut off) (Merck KGaA) at $4000 \times g$ at $4^{\circ} \mathrm{C}$ and was further purified by Superdex 200 10/200GL (Cytiva, Marlborough, MA, USA) size-exclusion chromatography column equilibrated with $20 \mathrm{mM}$ HEPES-NaOH, $\mathrm{pH}$ 7.5, $1 \mathrm{M} \mathrm{NaCl}$. 
Expression and purification of hFGFR1. pcDNA-SecreconAA-hFGFR1 (142-356)-His (50 $\mu \mathrm{g})$ was transfected to $3 \times 10^{6}$ Expi $293 \mathrm{~F}$ cells, and hFGFR1 proteins were expressed using the Expi293 Expression System (Thermo Fisher Scientific) according to the manufacturer's protocol. The culture medium $(60 \mathrm{~mL})$ that contained secreted hFGFR1 was dialyzed in $3 \mathrm{~L}$ of lysis buffer for $17 \mathrm{~h}$ at $4^{\circ} \mathrm{C}$. After dialysis, the medium was mixed with $500 \mu \mathrm{L}$ of Ni-NTA superflow resin using a rotator for $1 \mathrm{~h}$ at $4^{\circ} \mathrm{C}$. The resin was transferred into the disposable column and washed with $60 \mathrm{~mL}$ of wash buffer. hFGFR1 proteins were eluted with $3 \mathrm{~mL}$ elution buffer from the Ni-NTA resin, and the eluted fraction was diluted with two volumes of TBS. Diluted hFGFR1 was concentrated using Amicon Ultra 4 centrifugal filter devices and further purified using Superdex 200 10/200GL size-exclusion chromatography column equilibrated with TBS.

SELEX. The DNA template for the initial pool was 5'-GGGTGTTAGCTGTTAGTATC-40 N-GGTACG ATCAGCTAGCCCTATAGTGAGTCGTATTA -3' (GeneDesign, Inc., Osaka, Japan), where the T7 promoter sequence is underlined and $40 \mathrm{~N}$ indicates 40 nucleotides (nt) of randomized sequence. Primers 1 and 2 were 5'-TAATACGACTCACTATAGGGCTAGCTGATCGTACC-3', 5'- GGGTGTTAGCTGTTAGTATC-3', respectively (Hokkaido System Science Co., Ltd., Sapporo, Japan). The pool of RNAs with 2'-fluoropyrimidine modifications was created by in vitro transcription of the randomized DNA template using the Y639F mutant T7 RNA polymerase ${ }^{44}$ to incorporate 2 '-fluoro modified pyrimidines. SELEX procedure against hFGF5 (21-242)His was performed as previously described with minor modification ${ }^{25}$ Briefly, RNA was incubated with resinimmobilized hFGF5 (21-242)-His in binding buffer $(20 \mathrm{mM}$ Tris $\mathrm{HCl} \mathrm{pH} \mathrm{7.4,} 200 \mathrm{mM}$ sodium chloride, $5 \mathrm{mM}$ magnesium chloride, $5 \mathrm{mM}$ calcium chloride, $0.05 \%$ TWEEN20, 10\% glycerol) supplemented with $10-100 \mu \mathrm{M}$ low-molecular-weight heparin (approximately $5000 \mathrm{Da}$ ) (Dalteparin sodium; Pfizer Inc., New York, NY, USA) and $0.1 \mathrm{mg} / \mathrm{mL}$ bovine serum albumin (Sigma-Aldrich Co. LLC.). After incubation, the resin was washed five to seven times with $1 \mathrm{~mL}$ of washing buffer $(20 \mathrm{mM}$ Tris $\mathrm{HCl} \mathrm{pH} \mathrm{7.4,500} \mathrm{mM} \mathrm{sodium} \mathrm{chloride,} 5 \mathrm{mM}$ magnesium chloride, $5 \mathrm{mM}$ calcium chloride, $0.05 \%$ TWEEN20, 10\% glycerol). Resins were finally washed three times with $1 \mathrm{~mL}$ of binding buffer supplemented with $3 \mathrm{M}$ urea, and four times with $1 \mathrm{~mL}$ of washing buffer. Tightly bound RNAs were eluted from the resin via the addition of $200 \mu \mathrm{L}$ of elution buffer $(20 \mathrm{mM}$ Tris $\mathrm{HCl} \mathrm{pH} 7.4$, $6 \mathrm{M}$ guanidinium chloride).

Aptamer preparation. F5f1, F5f3, F5f1_56, and F5f3_56 with 2'-fluoropyrimidine modifications were prepared as described previously ${ }^{25}$. Briefly, the templates of F5f1_56 and F5f3_56 were amplified from cloning vectors containing F5f1 and F5f3, respectively. All RNA samples were purified by denaturing polyacrylamide gel electrophoresis. RNA concentration was determined based on the molecular absorption coefficient at $260 \mathrm{~nm}$.

SPR experiments. SPR assays were performed as previously described using a BIAcore X instrument (Cytiva) with minor modification ${ }^{25}$. The $5^{\prime}$-biotinylated 16 mer poly dT16 oligo dissolved in SPR running buffer (20 mM Tris $\mathrm{HCl} \mathrm{pH} \mathrm{7.4,} 200 \mathrm{mM}$ sodium chloride, $5 \mathrm{mM}$ magnesium chloride, $5 \mathrm{mM}$ calcium chloride, $0.05 \%$ TWEEN20) was immobilized to both flow cell 1 and 2 on the surface of streptavidin sensor chip (Cytiva) at approximately 500 response units (RU). The aptamers containing a 16 mer poly A tail dissolved in SPR running buffer were immobilized to approximately $240 \mathrm{RU}$ in flow cell 2, utilizing the hybridization of the poly A-dT. Human FGF5 (R\&D Systems, Inc., Minneapolis, MN, USA) with 4 equivalents of low-molecular-weight heparin (Dalteparin sodium; Pfizer Inc.) in SPR running buffer was injected for $60 \mathrm{~s}$ into flow cells 1 and 2 of the sensor chip and dissociated for $180 \mathrm{~s}$. The signal of flow cell 1 was subtracted from that of flow cell 2 to eliminate nonspecific interactions. Low-molecular-weight heparin was added to suppress nonspecific binding of FGF5 to the sensor chip. The sensorgrams were analyzed using BIA evaluation software (Cytiva). Dissociation constants were determined using a Langmuir (1:1) binding model. To assess the binding specificity of the aptamer, $100 \mathrm{nM}$ of recombinant human FGF1, FGF2, FGF4, FGF6, or FGFR1a (IIIc)-Fc chimera (all proteins purchased from R\&D Systems, Inc.) was injected with four equivalents of low-molecular-weight heparin.

For the competition assay, purified FGFR1 was dissolved in $20 \mathrm{mM}$ sodium acetate (pH 6.0) and immobilized to flow cell 2 on the surface of a CM5 sensor chip (Cytiva) by the amine coupling reaction at approximately 1800 RU. Then, 100 nM human FGF5 and 100 nM low-molecular-weight heparin in SPR running buffer were injected with or without $200 \mathrm{nM}$ aptamer. The signal of flow cell 1 was subtracted from that of flow cell 2 to eliminate nonspecific interactions.

Cell culture. Murine fibroblast NIH3T3 cell (RIKEN BRC) was grown in Dulbecco's Modified Eagle Media (DMEM) (FUJIFILM Wako Pure Chemical Corp.) supplemented with $10 \%$ fetal bovine serum (FBS, Thermo Fisher Scientific), 100 units/mL penicillin and $100 \mu \mathrm{g} / \mathrm{mL}$ streptomycin (FUJIFILM Wako Pure Chemical Corp.).

Cell proliferation assay without serum. In this assay, FBS was replaced with insulin after preculture of NIH3T3 cells to prevent the enzymatic degradation of aptamers by RNases that were spontaneously contained in FBS. Briefly, NIH3T3 cells were seeded at 5,000 cells/wells in a 96-well culture plate and precultured in DMEM with $10 \% \mathrm{FBS}$ at $37^{\circ} \mathrm{C}$ in $5 \% \mathrm{CO}_{2}$ for $24 \mathrm{~h}$. Cultured cells were washed twice with phosphate-buffered saline and exchanged in DMEM supplemented with $10 \mathrm{ng} / \mathrm{mL}$ insulin, $5 \mu \mathrm{g} / \mathrm{mL}$ heparin (Sigma-Aldrich Co. LLC.), and $100 \mathrm{ng} / \mathrm{mL}$ human FGF5 (R\&D Systems, Inc.) or $20 \mathrm{ng} / \mathrm{mL}$ human FGF2 (R\&D Systems, Inc. ). Then, 0-30 nM aptamer was added to each well, and cells incubated at $37^{\circ} \mathrm{C}$ in $5 \% \mathrm{CO}_{2}$ for $48 \mathrm{~h}$. Cells were counted by optical density (OD) at $450 \mathrm{~nm}$ using a WST-8 cell counting kit (Dojindo Molecular Technologies, Inc., Rockville, MD, USA). Inhibitory effect $\left(\mathrm{IC}_{50}\right)$ was calculated as following formula from the obtained $\mathrm{OD}_{450}$ values: 
$\%$ Inhibition $=100 \times\left[\mathrm{OD}_{450}\right.$ in the presence of FGF5)

- (OD 450 in the presence of FGF5 and each aptamer)]/

[(OD 450 in the presence of FGF2 $)-\left(\mathrm{OD}_{450}\right.$ in the absence of FGF2)]

The $\mathrm{IC}_{50}$ of each aptamer was determined by drawing an inhibition curve. The experiments were performed three times, and the mean and error were shown.

FGFR1 phosphorylation assay. NIH3T3 cells were cultured until semi-confluent growth was observed on a six-well plate, and the medium was replaced with serum-free DMEM. After incubation for $2 \mathrm{~h}$, cells were treated with $100 \mathrm{ng} / \mathrm{mL}$ FGF5 and $5 \mu \mathrm{g} / \mathrm{mL}$ heparin and 0 to $30 \mathrm{nM}$ aptamers for $1 \mathrm{~h}$ at $37^{\circ} \mathrm{C}, 5 \% \mathrm{CO}_{2}$. Cultured cells were washed and lysed on ice for $15 \mathrm{~min}$ with $100 \mu \mathrm{L}$ of RIPA buffer (Cell Signaling Technologies, Inc., Danvers, MA, USA) supplemented with protease and phosphatase inhibitor. Cell lysates were centrifuged at $21,500 \times g$ for $10 \mathrm{~min}$, and the supernatants were mixed with $4 \times$ Laemmli sample buffer (Bio-Rad Laboratories, Inc., Hercules, CA, USA). Cell lysate mixtures were subjected to SDS-PAGE and western blotting using antibodies to FGFR1 or phospho-FGFR1 (Cell Signaling Technologies, Inc.). The protein concentration was determined by the BCA assay.

Received: 21 August 2020; Accepted: 19 January 2021

Published online: 03 February 2021

\section{References}

1. Armelin, H. A. Pituitary extracts and steroid hormones in the control of 3 T3 cell growth. Proc. Natl. Acad. Sci. U. S. A. 70, 2702-2706 (1973).

2. Gospodarowicz, D. Localisation of a fibroblast growth factor and its effect alone and with hydrocortisone on 3T3 cell growth. Nature 249, 123-127 (1974)

3. 3Ornitz, D. M. \& Itoh, N. Fibroblast growth factors. Genome Biol. 2, doi:https://doi.org/10.1186/gb-2001-2-3-reviews3005 (2001).

4. 4Belov, A. A. \& Mohammadi, M. Molecular mechanisms of fibroblast growth factor signaling in physiology and pathology. Cold Spring Harb. Perspect. Biol. 5, doi:https://doi.org/10.1101/cshperspect.a015958 (2013).

5. 5Hui, Q., Jin, Z., Li, X., Liu, C. \& Wang, X. FGF Family: from drug development to clinical application. Int. J. Mol. Sci. 19, doi:https ://doi.org/10.3390/ijms19071875 (2018).

6. 6Zhang, X. et al. Receptor specificity of the fibroblast growth factor family. The complete mammalian FGF family. J. Biol. Chem. 281, 15694-15700 (2006).

7. Guillemot, F. \& Zimmer, C. From cradle to grave: the multiple roles of fibroblast growth factors in neural development. Neuron $71,574-588(2011)$.

8. Zhan, X., Bates, B., Hu, X. G. \& Goldfarb, M. The human FGF-5 oncogene encodes a novel protein related to fibroblast growth factors. Mol. Cell. Biol. 8, 3487-3495 (1988).

9. Zhan, X., Culpepper, A., Reddy, M., Loveless, J. \& Goldfarb, M. Human oncogenes detected by a defined medium culture assay. Oncogene 1, 369-376 (1987).

10. Hebert, J. M., Rosenquist, T., Gotz, J. \& Martin, G. R. FGF5 as a regulator of the hair growth cycle: evidence from targeted and spontaneous mutations. Cell 78, 1017-1025 (1994).

11. Higgins, C. A. et al. FGF5 is a crucial regulator of hair length in humans. Proc. Natl. Acad. Sci. U. S. A. 111, 10648-10653 (2014).

12. Suzuki, S., Ota, Y., Ozawa, K. \& Imamura, T. Dual-mode regulation of hair growth cycle by two Fgf-5 gene products. J. Invest. Dermatol. 114, 456-463 (2000).

13. Seo, H. R. et al. Intrinsic FGF2 and FGF5 promotes angiogenesis of human aortic endothelial cells in 3D microfluidic angiogenesis system. Sci. Rep. 6, 28832. https://doi.org/10.1038/srep28832 (2016).

14. Tian, R. et al. Fibroblast growth factor-5 promotes spermatogonial stem cell proliferation via ERK and AKT activation. Stem Cell. Res. Ther. 10, 40. https://doi.org/10.1186/s13287-019-1139-7 (2019).

15. Tuerk, C. \& Gold, L. Systematic evolution of ligands by exponential enrichment: RNA ligands to bacteriophage T4 DNA polymerase. Science 249, 505-510 (1990).

16. Ellington, A. D. \& Szostak, J. W. In vitro selection of RNA molecules that bind specific ligands. Nature 346, 818-822 (1990).

17. Nakamura, Y. Aptamers as therapeutic middle molecules. Biochimie 145, 22-33 (2018).

18. Jin, L., Nonaka, Y., Miyakawa, S., Fujiwara, M. \& Nakamura, Y. Dual therapeutic action of a neutralizing anti-FGF2 aptamer in bone disease and bone cancer pain. Mol. Ther. 24, 1974-1986 (2016).

19. Hamamoto, J. et al. The FGF2 aptamer inhibits the growth of FGF2-FGFR pathway driven lung cancer cells. Biochem. Biophys. Res. Commun. 503, 1330-1334 (2018).

20. Matsuda, Y. et al. Anti-angiogenic and anti-scarring dual action of an anti-fibroblast growth factor 2 aptamer in animal models of retinal disease. Mol Ther Nucleic Acids 17, 819-828 (2019).

21. Ito, C. et al. Decapeptide with fibroblast growth factor (FGF)-5 partial sequence inhibits hair growth suppressing activity of FGF-5. J. Cell. Physiol. 197, 272-283 (2003).

22. Sato, K., Hamada, M., Asai, K. \& Mituyama, T. CENTROIDFOLD: a web server for RNA secondary structure prediction. Nucleic Acids Res. 37, W277-280. https://doi.org/10.1093/nar/gkp367 (2009).

23. Clements, D. A., Wang, J. K., Dionne, C. A. \& Goldfarb, M. Activation of fibroblast growth factor (FGF) receptors by recombinant human FGF-5. Oncogene 8, 1311-1316 (1993).

24. Gelinas, A. D., Davies, D. R. \& Janjic, N. Embracing proteins: structural themes in aptamer-protein complexes. Curr. Opin. Struct. Biol. 36, 122-132 (2016).

25. Amano, R. et al. Kinetic and thermodynamic analyses of interaction between a high-affinity RNA aptamer and its target protein. Biochemistry 55, 6221-6229 (2016).

26. Takada, K. et al. Characterisation of an aptamer against the Runt domain of AML1 (RUNX1) by NMR and mutational analyses. FEBS Open Bio 8, 264-270 (2018).

27. Sakamoto, T., Ennifar, E. \& Nakamura, Y. Thermodynamic study of aptamers binding to their target proteins. Biochimie 145, 91-97 (2018). 
28. Jin, L. Aptamer for FGF2 and use thereof. Patent Application No. WO/2015/147017. https://patentscope2.wipo.int/search/en/detai 1.jsf?docId=WO2015147017 (2015).

29. Jurek, P. M. et al. Anti-FGFR1 aptamer-tagged superparamagnetic conjugates for anticancer hyperthermia therapy. Int. J. Nanomedicine 12, 2941-2950 (2017).

30. Ueki, R. et al. DNA aptamer assemblies as fibroblast growth factor mimics and their application in stem cell culture. Chem. Commun. (Camb.) 55, 2672-2675 (2019).

31. Kamatkar, N., Levy, M. \& Hebert, J. M. Development of a monomeric inhibitory RNA aptamer specific for FGFR3 that Acts as an activator when dimerized. Mol. Ther. Nucleic Acids 17, 530-539 (2019).

32. Kornmann, M., Ishiwata, T., Beger, H. G. \& Korc, M. Fibroblast growth factor-5 stimulates mitogenic signaling and is overexpressed in human pancreatic cancer: evidence for autocrine and paracrine actions. Oncogene 15, 1417-1424 (1997).

33. Hanada, K., Perry-Lalley, D. M., Ohnmacht, G. A., Bettinotti, M. P. \& Yang, J. C. Identification of fibroblast growth factor-5 as an overexpressed antigen in multiple human adenocarcinomas. Cancer Res. 61, 5511-5516 (2001).

34. Ghassemi, S. et al. FGF5 is expressed in melanoma and enhances malignancy in vitro and in vivo. Oncotarget 8, 87750-87762 (2017).

35. Zhou, Y. et al. Downregulation of fibroblast growth factor 5 inhibits cell growth and invasion of human nonsmall-cell lung cancer cells. J. Cell. Biochem. https://doi.org/10.1002/jcb.28107 (2018).

36. Han, D. et al. FGF5 promotes osteosarcoma cells proliferation via activating MAPK signaling pathway. Cancer Manag. Res. 11, 6457-6466 (2019).

37. Fang, F. et al. MicroRNA-188-5p suppresses tumor cell proliferation and metastasis by directly targeting FGF5 in hepatocellular carcinoma. J. Hepatol. 63, 874-885 (2015).

38. Liu, D. et al. MicroRNA-567 inhibits cell proliferation, migration and invasion by targeting FGF5 in osteosarcoma. EXCLIJ 17, 102-112 (2018).

39. Rosenquist, T. A. \& Martin, G. R. Fibroblast growth factor signalling in the hair growth cycle: expression of the fibroblast growth factor receptor and ligand genes in the murine hair follicle. Dev. Dyn. 205, 379-386 (1996).

40. Imamura, T. Physiological functions and underlying mechanisms of fibroblast growth factor (FGF) family members: recent findings and implications for their pharmacological application. Biol. Pharm. Bull. 37, 1081-1089 (2014).

41. Horiuchi, M., Takahasi, K., Kobashigawa, Y., Ochiai, M. \& Inagaki, F. A low-cost affinity purification system using beta-1,3-glucan recognition protein and curdlan beads. Protein Eng. Des. Sel. 25, 405-413 (2012).

42. Güler-Gane, G. et al. Overcoming the refractory expression of secreted recombinant proteins in mammalian cells through modification of the signal peptide and adjacent amino acids. PLOS ONE 11, e0155340. https://doi.org/10.1371/journal.pone.0155340 (2016).

43. García-Nafría, J., Watson, J. F. \& Greger, I. H. IVA cloning: A single-tube universal cloning system exploiting bacterial In Vivo Assembly. Sci. Rep. 6, 27459. https://doi.org/10.1038/srep27459 (2016).

44. Padilla, R. \& Sousa, R. A Y639F/H784A T7 RNA polymerase double mutant displays superior properties for synthesizing RNAs with non-canonical NTPs. Nucleic Acids Res. 30, e138. https://doi.org/10.1093/nar/gnf138 (2002).

\section{Acknowledgements}

The authors would like to thank Mrs. Kazumi Tomoda for helpful discussions and Enago (www.enago.jp) for the English language review.

\section{Author contributions}

T.S. and M.Y. designed and supervised the experiments. R.A., M.N., M.H., M.S., T.Y., Y.T., and F.I.G. performed the experiments and collected and analyzed the data. R.A., M.N., M.H., and T.S. wrote the manuscript. All authors reviewed the manuscript.

\section{Funding}

This research is supported by the Matching Planner Program (Grant Number MP28116808265) and Adaptable and SeamLess Technology transfer Program through Target-driven R\&D (A-STEP) (Grant Number VP30118067526) from Japan Science and Technology Agency, and JSPS KAKENHI (Grant Number 17 K05936 and 20K06529) from The Ministry of Education, Sports, Culture, Science and Technology (MEXT) of Japan.

\section{Competing interests}

The authors declare no competing interests.

\section{Additional information}

Supplementary Information The online version contains supplementary material available at https://doi. org/10.1038/s41598-021-82350-w.

Correspondence and requests for materials should be addressed to T.S.

Reprints and permissions information is available at www.nature.com/reprints.

Publisher's note Springer Nature remains neutral with regard to jurisdictional claims in published maps and institutional affiliations.

(c) (i) Open Access This article is licensed under a Creative Commons Attribution 4.0 International License, which permits use, sharing, adaptation, distribution and reproduction in any medium or format, as long as you give appropriate credit to the original author(s) and the source, provide a link to the Creative Commons licence, and indicate if changes were made. The images or other third party material in this article are included in the article's Creative Commons licence, unless indicated otherwise in a credit line to the material. If material is not included in the article's Creative Commons licence and your intended use is not permitted by statutory regulation or exceeds the permitted use, you will need to obtain permission directly from the copyright holder. To view a copy of this licence, visit http://creativecommons.org/licenses/by/4.0/.

(c) The Author(s) 2021 